\title{
An Unusual Paraneoplastic Syndrome of Synchronous Bladder Tumor and Prostate Cancer: Polymyositis
}

\author{
Hüseyin Badem¹, Mehmet Erol Yildirim² ${ }^{2}$ Serife Badem³ ${ }^{3}$ Özlem Şahin Balçık, \\ Sedat Tastemur ${ }^{1}$, Ersin Cimentepe ${ }^{2}$ \\ ${ }^{1}$ Urology Department, Turkey Yuksekihtisas Hospital, Ankara, Turkey \\ ${ }^{2}$ Urology Department, Turgut Ozal Universty Hospital, Ankara, Turkey \\ ${ }^{3}$ Radiology Department, Turkey Yuksekihtisas Hospital, Ankara, Turkey \\ ${ }^{4}$ Haematology Department, Turgut Ozal Universty Hospital, Ankara, Turkey \\ Email: huseyinbadem@yahoo.com
}

Received 29 June 2015; accepted 11 October 2015; published 14 October 2015

Copyright (C) 2015 by authors and Scientific Research Publishing Inc.

This work is licensed under the Creative Commons Attribution International License (CC BY). http://creativecommons.org/licenses/by/4.0/

(c) () Op Open Access

\section{Abstract}

Introduction: Polymyositis (PM) is a type of inflammatory myopathy that is associated with a broad range of malignant disorders. An association of PM with synchronous carcinoma of the bladder and prostate is extremely rare. Case: A 65-year-old man admitted to hematology with complaints of severe progressive weakness of lower extremities, hematuria and irritative urinary symptoms lasting for a month. The hemogram and erythrocyte sedimentation rate were normal. ALT was normal but AST was $405.56 \mathrm{U} / \mathrm{l}$. There was marked elevation of serum creatine kinase (CK) and lactate dehydrogenase, which were $14,065.15 \mathrm{U} / \mathrm{l}$ and $1267.50 \mathrm{U} / \mathrm{l}$, respectively. PSA was 4.28 and DRE was positive. The abdominal ultrasound revealed a $24 \times 20 \mathrm{~mm}$ soft tissue echogenicity lesion at the right wall and a $35 \times 21 \mathrm{~mm}$ soft tissue echogenicity lesion at the left wall of the bladder. The rest of the abdominal viscera were normal. Computed tomography found, two $6 \mathrm{~mm}$ solid lesions at left anterolateral and a $18 \times 12 \mathrm{~mm}$ solid lesion at inferoanterior bladder wall in addition to the above findings. Patient counseled to us. We resected all of the bladder masses with transurethral (TUR-BT) way and pathology revealed T2 high grade bladder tumor. Two days after TUR-BT, we performed a trans rectal ultrasonography guided prostate biopsy and pathology revealed a Gleason $3+4$ prostate cancer. EMG showed sensorimotor polyneuropathy at the lower extremities, sustaining polymyozitis. Biopsy of the right peroneus brevis muscle showed no vasculitis with low grade neurologic changes. We offered to perform a radical cystoprostatectomy operation but the patient prefered chemotherapy. Two months after his initial presentation at the second cure of the chemotherapy all muscle weaknesses showed a dramatic regression. Conclusion: This case report indicates that both bladder carcinoma and prostate cancer should be kept in mind in elderly PM patients presenting with lower urinary tract symptoms and hematuria. 


\section{Keywords}

\section{Polymyositis, Bladder Tumor, Prostate Cancer, Emg}

\section{Introduction}

Polymyositis (PM) is an idiopathic uncommon inflammatory myopathy characterized by pain and weakness in the proximal muscles, compatible electrophysiological and pathological findings [1]. If cutaneous symptoms like Gottron's sign and heliotrope erythema exist, we diagnose it as Dermatomyositis (DM). PM is often considered as a paraneoplastic syndrome because of three-fold higher incidence with cancer cases [2]. Even the PM is mostly associated with ovarian, lung, gastrointestinal cancers and non-Hodgkin's lymphoma, manifestation with the genitourinary cancers is rare [2]. We are herein reporting a case of PM as the first manifestation of the synchronous bladder tumor and prostate cancer

\section{Case}

A 65-year-old man admitted to hematology with complaints of severe progressive weakness of lower extremities, hematuria and irritative urinary symptoms lasting for a month. He had a 45 year history of cigarette smoking until three years ago. After hospitalization, the patient had counseled to rheumatology and the next laboratory tests were ordered by them. The common blood count and erythrocyte sedimentation rates were normal. ALT was normal even AST was $405.56 \mathrm{U} / \mathrm{l}$. There was marked elevation of serum creatinine kinase (CK) and lactate dehydrogenase (LDH), which were 14,065.15 U/l and $1267.50 \mathrm{U} /$, respectively. Prostate specific antigen (PSA) was $4.28 \mathrm{ng} / \mathrm{dl}$ and DRE was positive. Immunological tests (including antinuclear antibodies, anti-doublestranded DNA antibodies, anti-Sm, anti-Ro/SSA, anti-La/SSB, anti-RNP, anti-Jo 1 and complement levels except p-ANCA $=+++$ ), serum and urine protein electrophoresis and tumor markers showed negative results. The abdominal ultrasound revealed a $24 \times 20 \mathrm{~mm}$ soft tissue echogenicity lesion at the right wall and a $35 \times 21 \mathrm{~mm}$ soft tissue echogenicity lesion at the left wall of the bladder. The rest of the abdominal viscera were normal. Computed tomography found, two solid lesions at left anterolateral with a diameter of $6 \mathrm{~mm}$ and one solid lesion at inferoanterior wall of bladder with a diameter of $18 \times 12 \mathrm{~mm}$. Patient counseled to us. He had underwent transurethral resection of the tumors and pathology revealed T2 high grade bladder tumor. Two day after TUR-BT, we performed a transrectal ultrasonography guided prostate biopsy and pathology revealed a Gleason $3+4$ prostate cancer. EMG showed sensorimotor polyneuropathy at the lower extremities, sustaining Polymyositis. Biopsy of the right peroneus brevis muscle showed no vasculitis with low grade neurologic changes. We offered radical cystoprostatectomy operation but the patient preferred Gemcitabine + Cisplatin chemotherapy for both tumors. Two months after his initial presentation at the second cure of the chemotherapy all muscle weaknesses shown a dramatic regression. Also CK, LDH and AST levels were decreased to 55 U/I, 199 U/I and 13 U/I, respectively. After the end of the chemotherapy protocol, the patient decided to continue his follow up in another city, so that we could not get further information about the further treatment of his prostate cancer.

\section{Discussion}

Dermatomyositis is characterized by a pathognomonic heliotrope rash,Gottron papules (flat-topped, polygonal, and violaceous papules over the knuckles), and proximal muscle weakness [3]. Sometimes poikiloderma in a photosensitive distribution, periungual telangiectases, and facial erythema can be seen either [3].

The patient did not fulfill the cutaneous criteria for the diagnosis of DM [4]. Although he had the elevated CPK levels, proximal muscle weakness with the evidence of muscle biopsy and the EMG study results we diagnosed him as polymyositis (PM). There is a significantly more increased co-existence of ovary, lung and gastrointestinal cancers with DM should be the evidence of the paraneoplastic origin of the DM [5]. There are only few reports about the association of PM with bladder cancer or prostate cancer [6] [7], even there is no report about the existence of PM with the synchronous prostate and bladder cancer. Almog et al. reported a case of dermatomysitis seconder to the recurrent to bladder cancer [6]. In addition to that, de Sauza et al. reported a study of 12 dermatomyositis secondary to various malignancies of skin, gastrointestinal tract, prostate, thyroid, 
breast, lungs, and genitourinary tract [7]. Notwithstanding, the distinctive muscle features, long lasting lower urinary tract symptoms and the renowned association of PM with malignancy prompted an immediate workup, which indeed revealed the condition to be paraneoplastic. In most cases malignancy is diagnosed within 1 year of the onset of dermatomyositis [2].

However the pathogenesis of the paraneoplastic polymyositis (PM) is unclear, an immune-mediated mechanism is suspected [8]. Tumor antigens sharing antigenic determinants with muscle may trigger an immune response to these tissues [8]. Sometimes the diagnosis of bladder cancer or prostate cancer can be done subsequent that of PM.

There are some empirical therapies of PM like corticosteroids, azathioprine, cyclophosphamide, plasmapheresis and, more recently, intravenous gamma globulin [9]. But ablation of primary tumor showed regression of polymositis symptoms and serological manifestations [10]. Our patient showed a dramatically response to the chemotherapy. But we could not examine, which one was the main cause so as to the chemotherapy that the patient had treated have been for both tumors.

\section{Conclusion}

Both bladder carcinoma and prostate cancer should be kept in mind in elderly PM patients presenting with lower urinary tract symptoms and hematuria. Also, when symptoms of the paraneoplastic syndrome again increase, it may also detect recurrence and progression of the primary malignancy.

\section{References}

[1] Sabio, J.M., Vargas-Hitos, J.A. and Jiménez-Alonso, J. (2006) Paraneoplastic Dermatomyositis Associated with Bladder Cancer. Lupus, 15, 619-620. http://dx.doi.org/10.1177/0961203306071923

[2] Hill, C.L., Zhang, Y., Sigurgeirsson, B. and Pukkala, E. (2001) Frequency of Specific Cancer Types in Dermatomyositis and Polymyositis: A Population-Based Study. The Lancet, 357, 96-100.

http://dx.doi.org/10.1016/S0140-6736(00)03540-6

[3] Sagi, L., Amichai, B., Barzilai, A., Shpiro, D., Baum, S., Neimushin, A., et al. (2009) Dermatomyositis and Small Cell Carcinoma of the Bladder. Canadian Family Physician, 55, 997-999.

[4] Bohan, A. and Peter, J.B. (1975) Polymyositis and Dermatomyositis (First of Two Parts). The New England Journal of Medicine, 292, 344-347. http://dx.doi.org/10.1056/NEJM197502132920706

[5] Mallon, E., Osborne, G., Dinneen, M., Lane, R.J., Glaser, M. and Bunker, C.B. (1999) Dermatomyositis in Association with Transitional Cell Carcinoma of the Bladder. Clinical and Experimental Dermatology, 24, 94-96. http://dx.doi.org/10.1046/j.1365-2230.1999.00415.x

[6] Almog, Y., Ben Yehuda, A. and Ben Chetrit, E. (1991) Dermatomyositis Associated with the Recurrence of Transitional Cell Carcinoma and Kaposi's Sarcoma. Clinical and Experimental Rheumatology, 9, 285-288.

[7] de Souza, F.H. and Shinjo, S.K. (2012) Newly Diagnosed Dermatomyositis in the Elderly as Predictor of Malignancy. Revista Brasileira de Reumatologia, 52, 717-721.

[8] Alexander, S. and Forman, L. (1968) Dermatomyositis and Carcinoma. British Journal of Dermatology, 80, 86-96. http://dx.doi.org/10.1111/j.1365-2133.1968.tb12266.x

[9] Dalakas, M.C., Illa, I., Dambrosia, J.M., Soueidan, S.A., Stein, D.P., Otero, C., et al. (1993) A Controlled Trial of High-Dose Intravenous Immune Globulin Infusions as a Treatment for Dermatomyositis. The New England Journal of Medicine, 329, 1993-2000. http://dx.doi.org/10.1056/NEJM199312303292704

[10] Bouropoulos, C., Kanellakopoulou, K.D., Zarakovitis, I.E. and Melekos, M.D. (1997) Paraneoplastic Polymyositis Associated with Transitional Cell Carcinoma of the Bladder. The Journal of Urology, 157, 950-951. http://dx.doi.org/10.1016/S0022-5347(01)65095-X 